\title{
Soins en fin de vie: un souffle nouveau et des éléments pratiques précieux
}

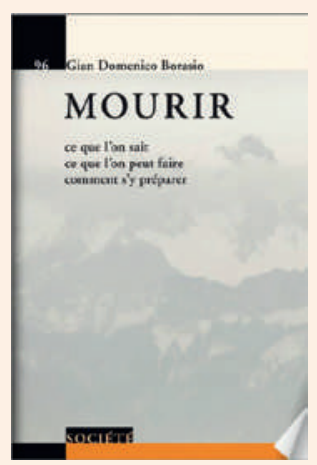

Gian Domenico Borasio Mourir

Ce que l'on sait, ce que l'on peut faire, comment s'y préparer Lausanne: Presses polytechniques et universitaires romandes; 2014.

155 pages. $17.50 \mathrm{CHF}$. ISBN 978-2-8891-5011-3
Dans la collection «Le Savoir suisse» paraît la traduction et adaptation au contexte suisse d'un ouvrage qui a vivement retenu l'attention en Allemagne [1]. Gian Domenico Borasio est chef du service de soins palliatifs du CHUV, à Lausanne, où il est arrivé de Munich en mars 2011 [2]. Son livre aborde de manière large les dimensions liées à la fin de vie dans notre société - médicales, physiques, psychosociales, spirituelles, juridiques - et les manières d'être meilleurs aujourd'hui à ces égards.

\section{La mort et le mourir}

Quelques rappels, d'abord quant au fait que la mort est un processus. «Aujourd'hui nous savons avec certitude que l'être humain ne meurt pas «d'un seul coup». Les organes s'épuisent à des vitesses et à des moments différents avant leur arrêt définitif.» A propos du sujet débattu de la mort cérébrale: «Elle représente le moment à partir duquel l'intégrité de l'organisme, qui exige au minimum le bon fonctionnement du tronc cérébral, est irréversiblement perdue.» Si les critères pertinents sont observés, elle est la mort, sans qu'un retour soit imaginable. Cela étant: «Comme souvent avec la fin de la vie, les problèmes que pose la mort cérébrale ne sont pas rationnels mais plutôt intuitifs et psychologiques. Il est simplement difficile d'accepter qu'une personne semblant dormir soit morte.»

\section{Attitudes médicales}

L'auteur évoque, élément qui tarde à perdre de son poids quotidien, la vexation narcissique que représente pour beaucoup le fait qu'un de ses patients meure. «Cette attitude de refus d'un échec est encore répandue et provoque des souffrance inutiles à bien des patients et leurs familles.» Et il rappelle la belle formule (attribuée à plusieurs auteurs): «Guérir parfois, soulager souvent, consoler toujours». A propos de fin de vie, on parle beaucoup de bienveillance médicale. «Ne confondons toutefois pas bienveillance avec paternalisme. La bienveillance ne revient pas à décider pour son patient mais à l'aider à prendre lui-même la décision la plus appropriée» [3].

Parmi les angoisses les plus répandues, il y a la peur de mourir de soif et celle de mourir par étouffement. Sur ces points qui font encore débat - alors que cela ne devrait plus être le cas: «Presque automatiquement, médecins et soignants prescrivent du liquide par voie intraveineuse et de l'oxygène par voie nasale [...] Ces deux mesures sont a priori tout à fait bienveillantes mais présentent deux inconvénients majeurs: elles sont inutiles et elles nuisent au patient» (lire les pages 99 à 108 - y compris à propos de l'usage souhaitable de la morphine). Et aussi: «Toutes les études concluent que la pose d'une gastrostomie percutanée endoscopique (GPE) chez des patients déments sévères n'atteint aucun des objectifs visés. La médecine moderne doit chez eux bannir cette pratique.»

En général, cette notation: "Naissance et mort, deux processus parallèles: dans les deux cas, la nature fait d'autant mieux son travail que la médecine ne s'en mêle pas. Or, la réalité est tout autre. La médecine moderne intervient toujours plus souvent de façon toujours plus invasive et souvent inutile.» Même s'il se peut que l'auteur force le trait pour être mieux compris, le message mérite d'être gardé à l'esprit.

\section{Mettre en place des dispositifs suffisants de soins palliatifs}

L'interdisciplinarité est au cœur des soins palliatifs. «Cette évidence s'est d'emblée imposée à Cicely Saunders, fondatrice de cette médecine (qui crée le St Christopher's Hospice à Londres en 1967) qui aurait davantage mérité le Nobel de médecine que bon nombre de biologistes moléculaires. [Par ses plusieurs formations] Elle réunissait dans sa personne les trois professionsclés: infirmière, assistante sociale et médecin» - à quoi on peut ajouter le/la psychologue.

Dans des conditions optimales, «plus de $90 \%$ des personnes en fin de vie pourraient mourir en bénéficiant d'un bon accompagnement sans avoir jamais vu un médecin spécialiste de soins palliatifs. C'est possible à condition que tous les praticiens aient acquis les connaissances nécessaires.»

Selon Borasio, la Suisse est tout à fait en mesure de développer une offre médicale en fin de vie de haute qualité. Le chemin a été balisé par la récente Stratégie nationale de soins palliatifs. Il y a pourtant «toujours un risque que des acteurs défendant des intérêts particuliers freinent le processus.» Comme partout dans le domaine de la santé relève-t-il, les soins optimaux peuvent être limités par des préoccupations sectorielles.

\section{L'importance maintenant reconnue de} l'assistance spirituelle - Du rôle des aumôniers «Pour la première fois dans l'histoire de la médecine moderne, la définition d'une discipline place les problèmes psychosociaux et spirituels au même niveau.» Est citée la définition de l'OMS des soins palliatifs, qui «cherchent à améliorer la qualité de vie des patients et de leur famille par la prévention et le soulage- 
ment de la souffrance et des autres problèmes physiques, psychologiques et spirituels qui lui sont liés.» Il y a peu encore, souvent on ne prêtait guère attention dans les hôpitaux au service d'aumônerie. «La communication avec les aumôniers était à peu près nulle. Le climat a changé [...] Aujourd'hui les Eglises délèguent leurs meilleurs pasteurs dans les hôpitaux. Et alors que l'œcuménisme en général a tendance à stagner, les expériences de coopération en milieu hospitalier ont la cote» [4].

Les aumôniers d'hôpitaux (si adéquatement formés) effectuent un travail impressionnant selon le prof. Borasio. «L'état de conscience limité d'un patient ne les empêche pas d'accomplir leur mission. Ils arrivent à se mettre à la place de malades dont il n'y a plus rien à attendres. Cette faculté d'empathie est très utile lorsque la décision relative au traitement est difficile à prendre.»

Le premier poste européen de professeur en assistance spirituelle au sein d'une Faculté de médecine a été créé en 2010 à Munich. «Non sans difficultés: un professeur au sommet de la hiérarchie a demandé sans plaisanter quelle était la différence entre l'assistance spirituelle et l'aromathérapie, témoignant de la difficulté à reconnaître comme équivalentes d'autres compétences que les siennes.»

\section{Le patient}

Il est au centre. Borasio lui donne une liste de 12 conseils pour réussir l'entretien avec son médecin, parmi lesquels: 1 . Réfléchissez si une personne de confiance devrait vous accompagner; [...] 6. Commencez par raconter au médecin - s'il ne vous le demande pas spontanément - ce que vous savez, pensez ou supposez déjà; 7. Parlez de vos peurs, de vos espoirs et de vos craintes; 8 . Posez tout de suite une question si vous ne comprenez pas; [...] 10. Demandez au médecin d'expliciter toutes les alternatives de la stratégie thérapeutique qu'il recommande [...] En cas de maladie très grave, demandez-lui si un traitement exclusivement palliatif ne serait pas aussi une bonne alternative.

Les attitudes et sentiments de malades terminaux ne sont pas ce qu'on imaginerait: «Dans le contexte de la fin de vie, on observe chez tous les patients d'une recherche de Martin Fegg (2005) un déplacement des valeurs égoïstes vers les valeurs altruistes. Le résultat se vérifie indépendamment de la religion ou du type de maladie. Le contraste avec les scores obtenus pour les personnes en bonne santé est impressionnant» [5].

\section{Directives anticipées et valeurs personnelles}

Le chapitre 8 de l'ouvrage parle des dispositions souhaitables pour sa fin de vie, notamment les directives anticipées (DA). Présentant les règles qui valent en Suisse depuis l'entrée en vigueur du nouveau droit de protection de l'adulte en 2013. Nous devrions tous envisager de rédiger des DA. G.D. Borasio insiste sur l'importance dans ce cadre de réfléchir à et formuler nos valeurs personnelles principales (voir p. 114-118).
Au chapitre 9 sont traitées la question de l'euthanasie et, particulière à la Suisse, celle de l'assistance au suicide (dont on rappelle qu'elle ne peut faire l'objet de DA). On salue le caractère pondéré, non idéologique, de ses positions, contributions précieuses à des débats parfois difficiles - et qui vont le rester; on pense à l'intention d'Exit d'ouvrir ses prestations aux personnes «fatiguées de la vie». A relever que l'auteur discute aussi les interrogations autour des expériences de mort imminente.

\section{Aujourd'hui et demain - Promouvoir largement les soins palliatifs}

Bien que conscient de certains conservatismes, celui qui rédige ces lignes est interpellé par les craintes de freinage ou diversion que relève G.D. Borasio: «L'essor de la médecine palliative a déjà réussi à initier un changement de perspective, d'une médecine technocentrique à une médecine plus holistique. Néanmoins elle se heurte à des résistances considérables.» On veut croire que ses collègues et partenaires, à la Faculté, à l'hôpital et ailleurs, reconnaissent le bien-fondé d'objectifs et de méthodes différents quand on parle de lutte contre la souffrance irréversible. Quand la guérison n'est plus une option réaliste, la priorité (qui n'a besoin d'aucune justification particulière!) est de promouvoir la meilleure qualité de vie possible. Etant rappelé que les travaux scientifiques disponibles montrent que les soins palliatifs, tout en se distançant de l'obstination thérapeutique, permettent en réalité de prolonger la vie plutôt qu'ils ne la raccourcissent - et qu'ils le font dans des conditions, en particulier de relation humaine, beaucoup meilleures.

Ce livre est une présentation substantielle, basée sur une grande expérience clinique et humaine. Lecture hautement recommandée pour les professionnels de la santé comme d'autres domaines (psychologie, travail social, aumônerie, droit). C'est aussi une source précieuse pour qui souhaite aborder, et aborder à temps, soi-même et dans les relations avec son entourage, les questions que pose notre inéluctable finitude.

\section{Références}

1 Borasio GD. Über das Sterben. München: Verlag C. H. Beck; 2012.

2 Martin J. Ne pas cantonner les soins palliatifs dans un coin, à distance de la «médecine qui guérit». Bull Méd Suisses. 2011;92(47):1846. Publié en allemand sous le titre «Palliativmedizin darf nicht in eine Ecke verbannt werden, weit weg von der «Medizin, die heilt - même réf.

3 Avec cette recommandation: dans l'information et le dialogue, «toujours commencer où est le patient».

4 Dans la pratique, avec un sourire: A l'idée d'un éventuel entretien avec les aumôniers, les patients s'exclament souvent «Oh, vous savez, je ne suis pas très religieux!» Ce à quoi nous répliquons: «Notre aumônier non plus».

5 Noter aussi que Borasio consacre son chapitre 5 à un thème qui prend de l'actualité, l'utilité possible pour les patients de techniques de méditation. 